\title{
The PROSA PDF fit and prompt neutrino fluxes
}

\author{
Maria Vittoria Garzelli for the PROSA collaboration*† \\ University of Hamburg, II Institute for Theoretical Physics \\ E-mail: maria.vittoria.garzelliedesy.de
}

Prompt neutrino fluxes are background for searches of astrophysical neutrinos at Very Large Volume Neutrino Telescopes. We present predictions for these fluxes, obtained by state-of-the-art QCD methods, and we discuss their uncertainties, in particular those related to our approximate knowledge of Parton Distribution Functions. We make use of the PROSA fit, the first fit appeared in literature including LHCb data on heavy-meson hadroproduction, specifically designed for low- $x$ physics. We present the present status of the fit and prospects for future developments. We compare our predictions with experimental results from the IceCube collaboration.

The European Physical Society Conference on High Energy Physics

5-12 July, 2017

Venice, Italy

\footnotetext{
* Speaker.

${ }^{\dagger}$ Members of the PROSA collaboration actively participating to this work include: O. Zenaiev, S. Moch, A. CooperSarkar, A. Geiser, K. Lipka, R. Placakyte and G. Sigl.
} 


\section{Introduction}

Prompt neutrino fluxes arising from the decays of heavy-hadrons produced by the interaction of ultra-high-energy (UHE) cosmic rays (CRs) with the Earth atmosphere, are a background for searches of high-energy neutrinos at Very Large Volume Neutrino Telescopes (VLV $v$ Ts), like IceCube and KM3NeT [1]. In particular, IceCube has already observed astrophysical neutrinos up to energies of some PeV. Prompt neutrinos of these energies are tipically generated by the decay of heavy-hadrons produced in $p p$ collisions at center-of-mass energies $E_{C M}$ not larger than those already reached at the Large Hadron Collider (LHC). However, while most of the data collected at LHC refer to the emission of particles at large transverse momenta $p_{T}$, the production of prompt neutrinos in the atmosphere covers a wider phase-space area, including also the forward direction corresponding to large rapidities. Additionally, UHE CRs can reach energies well beyond the LHC ones, meaning that the spectrum of prompt neutrino fluxes extend well beyond the $\mathscr{O}(\mathrm{PeV})$ energy range. One of the uncertainties stemming from the lack of experimental $p p$, ep collider and fixed-target data at very-high energies, with consequences on the predictions of high-energy prompt neutrino fluxes, is our uncertain knowledge of parton distribution functions (PDF) of protons and nuclei. In particular, the various data traditionally included in PDF fits, have allowed to gain important knowledges on PDFs down to longitudinal momentum fractions of the order of $x \sim$ $10^{-4}$ [2]. However, the higher is the $E_{C M}$ of a $p p$ collision and the measured rapidity of the emitted particles, the lower may be the $x$ values probed. In case of collisions initiated by CRs with energies in the tail of the primary CR spectrum, $x$ values smaller than $10^{-4}$ are tipically probed. The PROSA PDF fit is the first fit which has allowed to reach a better control of PDFs at low $x$ 's, i.e. for $x$ 's down to $\sim 10^{-6}$, thanks to the incorporation of LHCb data on heavy-quark hadroproduction at mid-rapidities. In this contribution we apply this fit to the computation of prompt neutrino fluxes and we compare PDF uncertainties to other uncertainties affecting these quantities.

\section{The PROSA PDFs and LHCb data on heavy-meson hadroproduction}

In the following we briefly describe the PROSA PDF fit, first proposed in Ref. [3], where more detail can be found. This fit takes into account all HERA data used for the HERAPDF1.0 PDF fit [4], i.e. neutral current and charged current inclusive deep-inelastic-scattering (DIS) combined data, $c \bar{c}$ DIS combined data and $b \bar{b}$ DIS ZEUS data, together with data on charmed hadron and bottom meson hadroproduction in the $p_{T}$ range $0<p_{T, c}<8 \mathrm{GeV}, 0<p_{T, b}<40 \mathrm{GeV}$ and in the rapidity range $2<y<4.5$, as published by the LHCb collaboration in Ref. [5] and [6], respectively. Three variants of the PROSA PDF fit exist, one including only the HERA data, another one which includes also LHCb absolute differential cross-sections in the aforementioned kinematic range, and finally a last one where for each fixed $\mathrm{LHCb} p_{T, \text { had }}$ bin, the ratios of distributions $(d \sigma / d y) /\left(d \sigma / d y_{0}\right)$ in different rapidity bins (i.e. normalized to the central bin $3<y_{0}<3.5$ ) are considered. The last variant is characterized by the lowest uncertainties, because theoretical uncertainties of QCD origin partly cancel in these ratios. In the following, we present predictions obtained by using as input the last variant of the PROSA PDF fit. The next-to-leading order fit has been performed in the fixed-flavour-number scheme, with 3 active flavours, a proper configuration for the description of the experimental data at low $p_{T, h a d}$. The theoretical description of 
the production of heavy hadrons adopted in the fit is based on the collinear factorization framework, with hard-scattering matrix-element computed with the MNR code [7], complemented by phenomenological Fragmentation Functions and Fragmentation Fractions [8].

After the publication of Ref. [3], LHCb results on $D$-meson hadroproduction at $\sqrt{s}=13 \mathrm{TeV}$ and $5 \mathrm{TeV}$ appeared in Ref. [9] and [10], respectively. During spring 2017, the LHCb collaboration has provided a new version of these works, with important revisions/errata of the already published data. In Fig. 1 we present an illustrative example of comparison of our theoretical predictions for $D^{ \pm}$hadroproduction, obtained with the POWHEG-BOX [11] + PYTHIA [12] event generator, using as input the PROSA PDFs, with the experimental data at $\sqrt{s}=13 \mathrm{TeV}$, after the last revision of the latter. The experimental data, not included in the PROSA PDF fit, turn out to lie within theoretical uncertainties in all considered bins. Theoretical uncertainties are dominated by factorization and renormalization $\left(\mu_{R}\right.$ and $\left.\mu_{F}\right)$ scale uncertainties, whereas PROSA PDF uncertainties, together with uncertainties on the charm pole mass $m_{c}$, also shown in the plots, are much smaller. The smallness of PDF uncertainties, especially in the large rapidity bins, is a consequence of the incorporation of the LHCb data into the PROSA fit. When considering other PDF fits which do not include these data, the uncertainties may be larger, as shown e.g. in Ref. [13].

\section{Prompt neutrino fluxes with PROSA PDFs}

Prompt neutrino fluxes have been computed according to the methodology described in Ref [14, 1], using as input the PROSA PDF fit and accounting for the production and decay of $D^{ \pm}, D^{0}, \bar{D}^{0}$, $D_{s}^{ \pm}$and $\Lambda_{c}^{ \pm}$. Predictions are shown in Fig. 2, using as input two different primary CR all-nucleon spectra. While the characteristic features of the all-particle primary spectrum of the CRs reaching the Earth atmosphere are well known and have been measured by several experiments, many uncertainties exist in the transformation to an all-nucleon spectrum, reflecting the uncertainties on the primary CR composition, especially evident at the highest energies, where a direct measurements of CR properties is not possible. The specific shapes of the distributions of prompt neutrino fluxes shown reflect the shape of the all-nucleon spectra, characterized by three different components/populations, supposed to be of different astrophysical origin. QCD uncertainties are also shown in Fig. 2. Prompt neutrino fluxes, as charm hadroproduction (see Fig. 1), turn out to be dominated by $\mu_{F}$ and $\mu_{R}$ scale uncertainties. The PROSA PDF uncertainties increase with energy, but turn out to be smaller than scale uncertainties even for energies $E_{v, l a b}$ well above the $\mathscr{O}(\mathrm{PeV})$.

Finally, in Fig. 3 our predictions are compared with the IceCube upper limit on prompt neutrino fluxes at $90 \%$ C.L. obtained in a six-year analysis of muon track data from the Northern Emisphere [16]. The Icecube limit turns out to lie just slightly above the central predictions for the prompt $\left(v_{\mu}+\bar{v}_{\mu}\right)$ flux obtained in this study, but well inside our global QCD uncertainty band. This holds over the entire range of relevant neutrino energies and challenges the model assumptions on atmospheric fluxes at high-energies adopted in the IceCube analyses.

\section{Conclusions}

The PROSA PDF fit was the first PDF fit exploiting LHCb data on charm and bottom meson hadroproduction at $\sqrt{s}=7 \mathrm{TeV}$, in order to reduce PDF uncertainties in the $10^{-6}<x<10^{-4}$ 

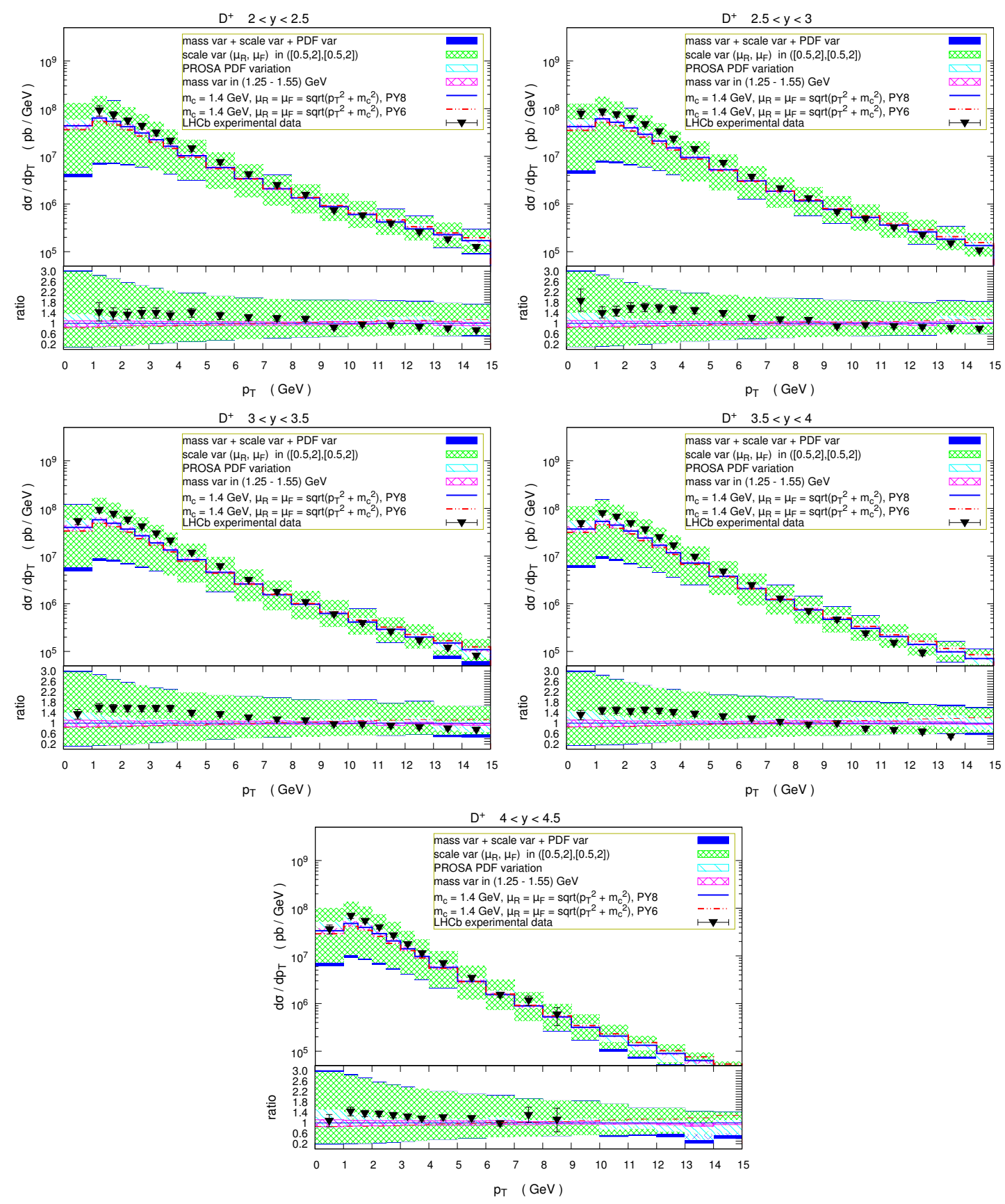

Figure 1: Transverse momentum distribution of $D^{ \pm}$mesons in $p p$ collisions at $\sqrt{s}=13 \mathrm{TeV}$. POWHEG-BOX + PYTHIA8 (blue solid line)/PYTHIA6 (red dotted line) predictions for $\mu_{R}=\mu_{F}=\mu_{0}=$ $\sqrt{p_{T, c}^{2}+m_{c}^{2}}, m_{c}=1.4 \mathrm{GeV}$ and with the PROSA PDFs, are compared to LHCb experimental data [5] in different rapidity bins. Theoretical predictions are accompanied by their uncertainty bands, due to $\mu_{R}$ and $\mu_{F}$ scale variation (green), to $m_{c}$ (magenta) and to PROSA PDF (light-blue hatched) variation. The LHCb experimental data [5] are shown together with their statistical and systematic uncertainties, added in quadrature. In the lower panel, ratios of the uncertainties with respect to the theoretical central predictions are shown, together with the ratio of the central theoretical predictions obtained with POWHEGBOX + PYTHIA6 with respect to those with POWHEG-BOX + PYTHIA8, and the ratio of experimental data with respect to theoretical predictions by POWHEG-BOX + PYTHIA8. 

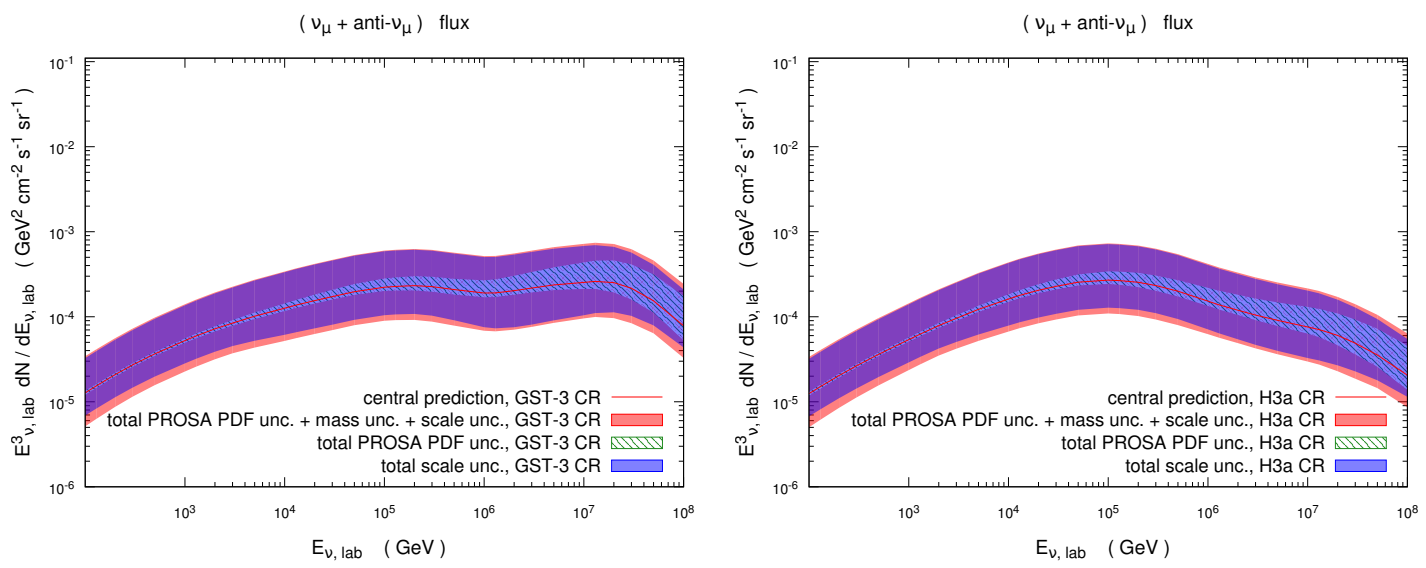

Figure 2: Central predictions for the prompt $\left(v_{\mu}+\bar{v}_{\mu}\right)$ flux together with their QCD uncertainties, computed by means of POWHEGBOX + PYTHIA 8 , as a function of the neutrino energy $E_{v, l a b}$. The uncertainty contributions due to $\mu_{R}$ and $\mu_{F}$ scale variation and the PDF eigenvalues within the PROSA fit, are shown separately by bands of different styles and colors, together with the combination in quadrature of scale, PDF and charm pole mass uncertanties. Two different cosmic ray all-nucleon spectra, with an heavy composition at the highest energies, are used as input: the left panel corresponds to the GST-3 fit whereas the right panel corresponds to the H3a fit, both described in Ref. [15].

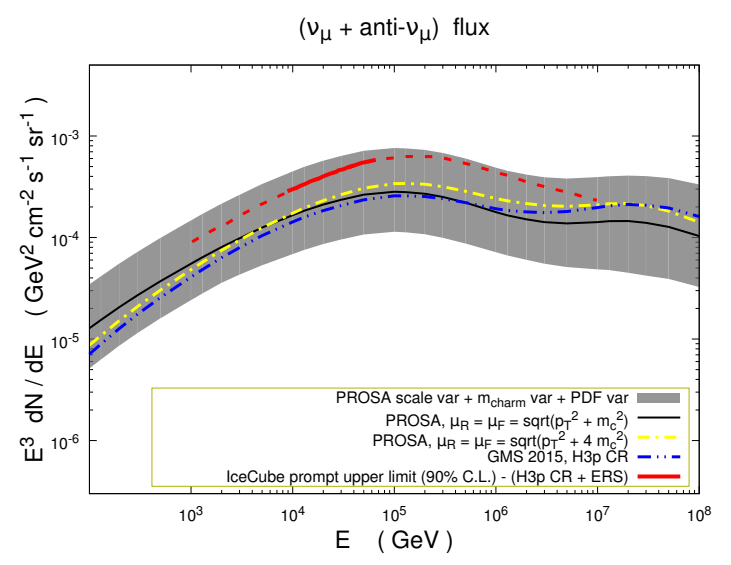

Figure 3: Comparison of the prompt $\left(v_{\mu}+\bar{v}_{\mu}\right)$ flux using the PROSA PDFs with the present upper limit on prompt neutrino flux at $90 \%$ confidence level obtained by the IceCube experiment [16] (solid red line) and its extrapolation to other energies (dotted red line), which adopted the ERS model [17] as a basis for modelling prompt neutrinos. Central predictions using the scale $\mu_{R}=\mu_{F}=\sqrt{p_{T}^{2}+4 m_{c}^{2}}$ and PROSA PDFs and ABM PDFs (GMS 2015) are also shown [14]. The limit and all predictions refer to the H3p CR flux [15].

range. Work is ongoing in order to further reduce the uncertainties affecting the PROSA PDF fit, by incorporating $\mathrm{LHCb}$ open charm data at 13 and $5 \mathrm{TeV}$, and the ratios of these data at different energies (13/5 and 13/7), which have already been considered in Ref. [18]. The PROSA PDF fit has been used as input for astrophysical applications. Here we have shown that, when considering the present PROSA fit, PDF uncertainties are not the dominant QCD uncertainty affecting prompt neutrino fluxes at the energies of interest for $\operatorname{VLV} v \mathrm{~T}$ analyses. 


\section{References}

[1] PROSA Collaboration, M. V. Garzelli, S. Moch, O. Zenaiev, A. Cooper-Sarkar, A. Geiser, K. Lipka, R. Placakyte, and G. Sigl, Prompt neutrino fluxes in the atmosphere with PROSA parton distribution functions, JHEP 05 (2017) 004, [arXiv: 1611 . 03815].

[2] A. Accardi et al., A Critical Appraisal and Evaluation of Modern PDFs, Eur. Phys. J. C76 (2016), no. 8 471, [arXiv:1603.08906].

[3] PROSA Collaboration, O. Zenaiev et al., Impact of heavy-flavour production cross sections measured by the LHCb experiment on parton distribution functions at low x, Eur. Phys. J. $\mathbf{C 7 5}$ (2015), no. 8 396, [arXiv:1503.04581].

[4] ZEUS, H1 Collaboration, F. D. Aaron et al., Combined Measurement and QCD Analysis of the Inclusive $e^{ \pm} p$ Scattering Cross Sections at HERA, JHEP 01 (2010) 109, [arXiv: 0911.0884 ].

[5] LHCb Collaboration, R. Aaij et al., Prompt charm production in pp collisions at $\sqrt{\mathrm{s}}=7 \mathrm{TeV}$, Nucl. Phys. B871 (2013) 1-20, [arXiv:1302.2864].

[6] LHCb Collaboration, R. Aaij et al., Measurement of B meson production cross-sections in proton-proton collisions at $\sqrt{s}=7 \mathrm{TeV}$, JHEP 08 (2013) 117, [arXiv: 1306.3663 ].

[7] M. L. Mangano, P. Nason, and G. Ridolfi, Heavy quark correlations in hadron collisions at next-to-leading order, Nucl. Phys. B373 (1992) 295-345.

[8] M. Lisovyi, A. Verbytskyi, and O. Zenaiev, Combined analysis of charm-quark fragmentation-fraction measurements, Eur. Phys. J. C76 (2016), no. 7 397, [arXiv: 1509.01061 ].

[9] LHCb Collaboration, R. Aaij et al., Measurements of prompt charm production cross-sections in pp collisions at $\sqrt{s}=13 \mathrm{TeV}$, JHEP 03 (2016) 159, [arXiv: 1510.01707 ]. [Erratum: JHEP05,074(2017)].

[10] LHCb Collaboration, R. Aaij et al., Measurements of prompt charm production cross-sections in pp collisions at $\sqrt{s}=5 \mathrm{TeV}, \mathrm{JHEP} 06$ (2017) 147, [arXiv: 1610.02230$].$

[11] S. Alioli, P. Nason, C. Oleari, and E. Re, A general framework for implementing NLO calculations in shower Monte Carlo programs: the POWHEG BOX, JHEP 1006 (2010) 043, [arXiv : 1002.2581 ].

[12] T. Sjostrand, S. Mrenna, and P. Z. Skands, A Brief Introduction to PYTHIA 8.1, Comput. Phys. Commun. 178 (2008) 852-867, [arXiv:0710 . 3820].

[13] M. Benzke, M. V. Garzelli, B. Kniehl, G. Kramer, S. Moch, and G. Sigl, Prompt neutrinos from atmospheric charm in the general-mass variable-flavor-number scheme, arXiv:1705.10386.

[14] M. V. Garzelli, S. Moch, and G. Sigl, Lepton fluxes from atmospheric charm revisited, JHEP 10 (2015) 115, [arXiv:1507.01570].

[15] T. K. Gaisser, T. Stanev, and S. Tilav, Cosmic Ray Energy Spectrum from Measurements of Air Showers, Front.Phys.China 8 (2013) 748-758, [arXiv: 1303 . 3565].

[16] IceCube Collaboration, M. G. Aartsen et al., Observation and Characterization of a Cosmic Muon Neutrino Flux from the Northern Hemisphere using six years of IceCube data, arXiv:1607.08006.

[17] R. Enberg, M. H. Reno, and I. Sarcevic, Prompt neutrino fluxes from atmospheric charm, Phys. Rev. D78 (2008) 043005, [arXiv: 0806.0418$].$

[18] R. Gauld and J. Rojo, Precision determination of the small-x gluon from charm production at LHCb, Phys. Rev. Lett. 118 (2017), no. 7 072001, [arXiv: 1610.09373 ]. 